\title{
28 Research Square \\ Structural Insights into the Binding of Nanobodies Lam2 and Lam4 to the Red Fluorescent Protein mCherry
}

\section{Ziying Wang}

Fudan University School of Life Sciences

\section{Long Li}

Fudan University

Rongting $\mathrm{Hu}$

Fudan University

\section{Peiyu Zhong}

Fudan University School of Life Sciences

\section{Yiran Zhang}

Fudan University

\section{Shihao Cheng}

Fudan University School of Life Sciences

\section{Rui Liu}

Fudan University School of Life Sciences

\section{Yu Ding ( $\nabla$ yuding@fudan.edu.cn )}

Fudan University https://orcid.org/0000-0001-7772-6449

\section{Research Article}

Keywords: nanobody, red fluorescent protein, mCherry, structure.

Posted Date: April 21st, 2021

DOl: https://doi.org/10.21203/rs.3.rs-424438/v1

License: @ (1) This work is licensed under a Creative Commons Attribution 4.0 International License. Read Full License 


\section{Abstract \\ Background}

Red fluorescent proteins (RFPs) are widely used in molecular biology research, especially in deep tissues and animal models, because of their superior autofluorescence, light scattering, and phototoxicity to GFP. Although RFP can be easily monitored in vivo, improved manipulation of RFP is still desired. Using suitable nanobodies (Nbs) to bind to different epitopes of RFP is the most promising approach; thus, it is crucial to obtain structural information on how the different Nbs interact with RFP.

\section{Results}

We determined the crystal structures of the LaM2-mCherry and LaM4-mCherry complexes at $1.4 \AA$ and $1.9 \AA$ resolution. Our results showed that LaM2 binds to the side of the mCherry $\beta$-barrel, while Lam4 binds to the bottom of the $\beta$-barrel and does not interfere with the homo-oligomerization interface. The distinct binding sites of LaM2 and LaM4 were further verified by ITC, F-SEC and DLS assays. Our results also showed that LaM2 and LaM4 can bind simultaneously to mCherry, which is crucial for recruiting multiple operation elements to the RFP. The binding of LaM2 or LaM4 did not significantly change the chromophore environment of mCherry, which is important for fluorescence quantification assays, while several GFP Nbs significantly altered the fluorescence. Mutation of the residues of the LaM2 or LaM4 binding interface to mCherry significantly decreased the binding affinity of the Nb to mCherry.

\section{Conclusions}

Our results provided atomic resolution interaction information on the binding of Nbs LaM2 and LaM4 binding with mCherry, which is important for developing detection and manipulation methods for RFP-based biotechnology.

\section{Introduction}

Fluorescent proteins (FPs) are the most extensively studied and widely used genetic tools in molecular biology research. Fluorescent proteins can be easily expressed in almost all kinds of cells, and the fusion of fluorescent proteins generally does not affect the function of other proteins. Compared to jellyfish-derived green fluorescent proteins, red fluorescent proteins (RFPs) are ideal for in vivo imaging due to their long-wavelength excitation and their lower light scattering, autofluorescence, and phototoxicity [1-3]. Thus, for transgenic RFP or RFP fusion proteins in small animal models or deep tissues, it is easy to monitor the RFP signal by imaging approaches due to the low background noise and deeper in vivo imaging of tissues and organs. Although many genetically encoded RFP animal strains have been established to facilitate live observation, the manipulation of RFPs will remain problematic until the discovery and development of RFP-specific nanobodies (Nbs) [4, 5].

Nbs, first discovered by Hamers Casterman in 1993 [6], are single domain antibodies derived from the heavy chain variable regions $(\mathrm{VHH})$ of Camelidae atypical immunoglobulins. Nbs are the smallest functional fragments derived from a naturally occurring immunoglobulin. Unlike monoclonal antibodies, Nbs can be easily produced in prokaryotic expression systems. Because of their small size $(12-15 \mathrm{kDa})$ and very high stability and solubility, Nbs are widely used for industrial [7] and clinical in vitro diagnostic (IVD) applications [8]. The small size also allows Nbs to be genetically encoded as chimera proteins and delivered to cells by fusion plasmids. Typically, the long CDR3 region 
enables $\mathrm{Nb}$ binding to an antigen with similar high specificity and affinity $[9,10]$, including binding with the smooth PD-L1 protein surface [11], inserting into canyons on the HIV envelope that are not accessible to IgG [12] to neutralize a broad range of HIV-1 strains, and effectively blocking the entry of SARS-CoV-2 spike protein [13-15].

Kirchhofer et al. first developed a series of GFP Nbs that can induce subtle opposing changes in the chromophore environment [16]. The GFP-specific Nbs GBP1 (GFP enhancer) and GBP4 (GFP minimizer) were suitable for monitoring protein expression, subcellular localization, and translocation. Our previous work also showed that the chimeric GFP Nb GFP-enhancer-(GGGGS)4-LaG16 increased the binding affinity of GFP and was suitable for GFPtagged target protein purification [17]. Tang et al. developed a GFP Nb-based system for the selective manipulation of diverse GFP-labeled cells across transgenic lines [18]. Later, Tang et al. achieved direct optogenetic control of GFP expression in neurons by Cre/loxP recombination through the binding of the GFP-specific Nb Cre chimera protein to GFP[19]. Herce et al. designed a cell-permeable $\mathrm{Nb}$ system to label and manipulate intracellular antigens in living cells[20]. Simpson performed PROTAC degradation of a GPF fusion protein with an anti-GFP Nb conjugated to the Halo-tag [21].

Although many GFP Nb-related protein visualization and manipulation applications have been introduced, few RFP $\mathrm{Nbs}$ have been reported. Fridy et al. generated a series of Nbs (named LaMs) that bind specifically to mCherry through a high-throughput screening method based on MS and cDNA libraries [22]. Prole and Taylor developed methods to visualize and manipulate intracellular signaling through GFP and GFP Nbs[23].

To develop an in vivo RFP manipulation system, the design of two or more Nbs fused with other manipulating components that can interact with different epitopes of the RFP surface at the same time is required. However, the lack of structural information on the detailed interaction interfaces between RFP and specific Nbs hinders the design and application of manipulation of RFP or RFP fusion proteins by high-affinity antibodies. Here, we determined the crystal structure of the LaM2-mCherry and LaM4-mCherry complexes and clarified the details of the binding of these two Nbs to mCherry. We also verified the simultaneous binding of LaM2 and LaM4 to RFP by a series of orthogonal molecular biology assays. Our results provide crucial atomic resolution interaction information for the further development of methods to manipulate RFP or RFP fusion proteins in vivo.

\section{Materials And Methods}

\section{Protein expression, purification and characterization}

The coding sequences of LaM2 and LaM4 were optimized based on favored codon usage in E. coli and were synthesized by Genewiz (Suzhou, China). For crystallization and binding assays, DNA encoding LaM2 and LaM4 was subcloned into the pET28a-SUMO vector with an N-terminal 6xHis tag followed by a SUMO tag or a pET21a-derived vector with an $\mathrm{N}$-terminal 10xHis tag, respectively. The plasmids were transformed into E. coli strain BL21 (DE3) for expression. The bacteria were cultured in LB medium at $37^{\circ} \mathrm{C}$ until the OD600 reached 0.8 . Recombinant protein expression was induced by the addition of $0.2 \mathrm{mM}$ isopropyl-D-1-thiogalactopyranoside (IPTG) and incubation for an additional $18 \mathrm{~h}$ at $18{ }^{\circ} \mathrm{C}$. The cells were harvested and resuspended in NiA buffer containing $20 \mathrm{mM}$ imidazole, 5\% glycerol, $150 \mathrm{mM} \mathrm{NaCl}$, and $100 \mathrm{mM}$ Tris-HCl, pH 7.5. The His10-tagged recombinant LaM2/LaM4 and their respective mutants were initially purified by Ni-NTA affinity purification using a HisTrap HP column (Qiagen) and eluted with $\mathrm{NiB}$ buffer containing $300 \mathrm{mM}$ imidazole, $5 \%$ glycerol, $150 \mathrm{mM} \mathrm{NaCl}$, and $100 \mathrm{mM}$ Tris pH 7.5. For crystallization, the His-SUMO tag was removed by incubation with recombinant ULP1 overnight at $4{ }^{\circ} \mathrm{C}$. The cleaved tag fragment and ULP1 were removed by passing through a HisTrap HP column. LaM2/LaM4 were further purified by size exclusion chromatography on a Superdex75 Increase column (Cytiva), and the buffer was exchanged to gel 
filtration buffer: $10 \mathrm{mM}$ HEPES, pH 7.4 and $100 \mathrm{mM} \mathrm{NaCl}$. The purity and molecular weight of the target proteins were verified by SDS-PAGE. Detailed information on the expression and purification of recombinant proteins is provided in the supplementary data.

\section{Site-directed mutagenesis}

Site-directed mutagenesis was carried out by employing a PCR-based mutagenesis site-directed method (Vazyme 2x Phanta Master Mix) using His10-LaM2 and His10-LaM4 as the template. The sequences of the primers used to generate these mutants are displayed in supplementary Table S1. All site-directed mutagenesis constructs were confirmed by DNA sequencing (RuiDi, Shanghai, China).

\section{Crystallization and data collection}

Concentrated LaM2 or LaM4 was mixed with mCherry at a molar ratio of 2:1 and incubated for $1 \mathrm{~h}$ at $4{ }^{\circ} \mathrm{C}$. The mCherry/LaM2 and mCherry/LaM4 complexes were separated from the excess LaM2/LaM4 by size exclusion chromatography using a Superdex75 Increase column (Cytiva). The mCherry/Nb complex was then concentrated to $15 \mathrm{mg} / \mathrm{ml}$ in gel filtration buffer containing $10 \mathrm{mM} \mathrm{HEPES}, \mathrm{pH} 7.4$ and $100 \mathrm{mM} \mathrm{NaCl}$. Crystals of the mCherry/LaM2 complex were obtained by the sitting-drop vapor diffusion method at $293 \mathrm{~K}$ in drops containing a mixture of $1 \mu$ l of protein solution and $1 \mu \mathrm{l}$ of reservoir solution, which consisted of $0.2 \mathrm{M}$ magnesium chloride hexahydrate, $0.1 \mathrm{M}$ Tris$\mathrm{HCl}, \mathrm{pH} 8.5,25 \% \mathrm{w} / \mathrm{v}$ polyethylene glycol 350. Crystals of the mCherry/LaM4 complex were obtained in $0.2 \mathrm{M}$ lithium sulfate monohydrate, $0.1 \mathrm{M}$ Bis-Tris, $\mathrm{pH} 5.5$, and $25 \% \mathrm{w} / \mathrm{v}$ polyethylene glycol 3350.

Cryoprotection was performed by adding glycerol to the reservoir buffer at a $20 \%$ concentration. X-ray diffraction data were collected at $100 \mathrm{~K}$ in beamlines BL17U1 [24] and BL19U1 [25], Shanghai Synchrotron Radiation Facility, Chinese Academy of Sciences.

\section{Determination and refinement of protein structure}

Diffraction images were indexed and processed by HKL2000 [26]. The structures of mCherry/LaM2 and mCherry/LaM4 were obtained by molecular replacement using the Phaser program from the CCP4 crystallography package [27] with mCherry (PDB ID: 2H5Q) and a GFP Nb (PDB ID: 3K1K) as the search model. Structure refinement was performed by Refmac [28] and Phenix [29]. The model was refined by COOT [30]. The crystallographic parameters of mCherry/LaM2 (PDB ID: 6IR2; $1.39 \AA$ ) and mCherry/LaM4 (PDB ID: 6IRI; $1.92 \AA$ ) are listed in Table 1. The related figures were drawn by PyMOL [31].

\section{Isothermal titration calorimetry (ITC)}

The thermodynamic parameters of the binding of LaM2/LaM4 and their respective mutants to mCherry were determined by ITC using a VP-ITC calorimeter (MicroCal VP-ITC, Malvern). In a typical experiment, each titration was performed by injecting a $12 \mu \mathrm{l}$ aliquot of protein sample $(0.15 \mathrm{mM}$ to $0.52 \mathrm{mM})$ into the cell containing another reactant $(0.015 \mathrm{mM}$ to $0.029 \mathrm{mM})$ at a time interval of $120 \mathrm{~s}$ to ensure that the titration peak returned to the baseline. Altogether, 23 aliquots were titrated in each individual experiment. The stoichiometry of binding $(n)$, the association constant $\mathrm{Ka}$, and the binding enthalpy $\mathrm{H}$ were evaluated using MicroCal Origin 7.0 software with a one-site binding model.

\section{Fluorescence-based Size Exclusion Chromatography (F-SEC)}


The oligomeric state of the tested samples in buffers were recorded by F-SEC. We used $100 \mu \mathrm{l} 0.1 \mathrm{mg} / \mathrm{ml} \mathrm{mCherry} \mathrm{as}$ control. For LaM2-mCherry and LaM4-mCherry complex, $50 \mu \mathrm{l} 0.2 \mathrm{mg} / \mathrm{ml}$ of mCherry (about $7 \mu \mathrm{M}$ ) and $0.2 \mathrm{mg} / \mathrm{ml}$ (about $14 \mu \mathrm{M}$ ) of LaM2 or LaM4 were mixed in equal volume (the final concentration of mCherry was $3.5 \mu \mathrm{M}$, and the final concentration of LaM2/LaM4 was $7 \mu \mathrm{M}$ ) and incubated on ice for 1 hour. For LaM2-LaM4-mCherry complex, 50

l $0.2 \mathrm{mg} / \mathrm{ml}$ mCherry (about $7 \mu \mathrm{M}$ ) was mixed with $25 \mu \mathrm{l} 0.4 \mathrm{mg} / \mathrm{ml} \mathrm{LaM} 2$ and $25 \mu \mathrm{l} 0.4 \mathrm{mg} / \mathrm{ml}$ LaM4 and incubated on ice for 1 hour. After high-speed centrifugation, the supernatants were loaded onto a Superdex 200 Increase sizeexclusion column (Cytiva) equilibrated with SEC buffer (20 mM HEPES pH 7.0, $150 \mathrm{mM} \mathrm{NaCl}$ ). The fluorescence of each sample was recorded by a fluorometer (excitation, $587 \mathrm{~nm}$; emission, $610 \mathrm{~nm}$ for mCherry fluorescence). The data were processed and normalized by FSECplotter software.

\section{Emission spectrum measurements}

The emission spectra of mCherry $(0.1 \mathrm{mg} / \mathrm{mL}$ ) and mCherry-LaM2/LaM4 (mCherry $0.1 \mathrm{mg} / \mathrm{mL}$ with excess Nb) were recorded using a fluorescence spectrophotometer (Varian Cary Eclipse). The excitation wavelength was $587 \mathrm{~nm}$. The emission spectrum was recorded between $550-700 \mathrm{~nm}$. The spectra data were analyzed with Origin.

\section{Dynamic light scattering assay}

The particle size distribution of mCherry, LaM2-mCherry complex, LaM4-mCherry complex and LaM2-LaM4-mCherry complex were measured by Nano-size-Zeta potential analyzer (Malvern Instruments, ZS90-2026), the test temperature is $25^{\circ} \mathrm{C}$, the test angle is 90 degrees.

\section{Results}

\section{The overall structure of the mCherry/LaM2 and mCherry/LaM4 complexes}

To gain insight into the binding sites of Nbs to RFPs, we purified recombinant LaM2, LaM4 and the RFP mCherry and then determined the crystal structures of the mCherry/LaM2 and mCherry/LaM4 complexes. The crystal of the mCherry complex contains mCherry and LaM2 or LaM4 at a 1:1 stoichiometry. The overall structure of mCherry/LaM2 was refined to $1.39 \AA ̊$ resolution, and that of mCherry/LaM4 was refined to $1.92 \AA$ resolution. The crystallographic data are shown in Table 1. The binding interface of CDRs 1-3 of LaM2/LaM4 and mCherry was well defined. LaM2-mCherry crystallized in the space group P $2{ }_{1} 2_{1} 2_{1}$, and the asymmetric unit contained one LaM2 $\mathrm{Nb}$ and one mCherry molecule. The Matthews coefficient was approximately $2.11 \AA^{3} / \mathrm{Da}$, and the solvent content was 41.58\%. LaM4-mCherry crystallized in the space group C121, and the asymmetric unit contained one LaM4 $\mathrm{Nb}$ and one mCherry molecule. The Matthews coefficient was approximately $2.14 \AA^{3} / \mathrm{Da}$, and the solvent content was $42.42 \%$.

Figure $1 \mathrm{~A}$ shows the overall structure of the LaM2-mCherry complex, and Figure 1B shows the overall structure of the LaM2-mCherry complex. The binding sites of LaM2 and LaM4 on mCherry were different. Figure 1C shows the superposed structures of LaM2-mCherry and LaM4-mCherry. LaM2 binds to the side of the $\beta$-barrel (the 4th and 5th $\beta$-sheets of the 11 total $\beta$-sheets), while LaM4 binds to the bottom of the $\beta$-barrel (both the amino and carboxyl termini of RFP are at the bottom). The binding modes of the Nbs themselves, the binding modes are also very different. Figure 1D compares the binding of LaM2 and LaM4. Although the Nb's constant domains are similar, the CDRs are totally different. CDR3 of Nb is longer than that in IgG, and therefore, while only a loop in the IgG secondary structure typically interacts with the antigen, an a-helix in the $\mathrm{Nb}$ may also emerge and provide an additional interaction mode with the antigen. CDR3 and CDR1 of LaM2 contain two a-helices: residues 123-126 (Ser-Glu-Asn- 
Asp) and residues 42-45 (Thr-Phe-Ser-Asp). CDR3 of LaM4 contains an a-helix consisting of residues 110-112 (GInArg-Leu). Additionally, the surface potentials of LaM2 and LaM4 are quite different; LaM2 has a large negative patch in CDR1 that contributes to salt bridge formation with mCherry, while the binding of LaM4 to mCherry does not involve a salt bridge (Figure 1E).

\section{Details of the binding sites of LaM2/LaM4 to mCherry}

Since the resolution of both Nb-mCherry complex crystals was high enough, the binding sites between LaM2/LaM4 and mCherry were clearly defined. The detailed interaction interfaces of LaM2 and LaM4 with mCherry are shown in Figure 2. In the LaM2-mCherry complex, all the CDRs 1-3 of LaM2 contributed to the binding to mCherry. In LaM2 CDR1, a hydrogen bond of $2.9 \AA$ A was formed between Ser44 and mCherry Lys92 and Lys182. In CDR2, there was a strong hydrogen bond between Ser66 and mCherry Glu94, a weak hydrogen bond between Ser68 and mCherry Glu94, and a weak hydrogen bond between His70 and mCherry Thr 180. In CDR3, the Trp119 and Tyr120 side chains were inserted in the pocket of mCherry, and a hydrogen bond formed between Tyr120 and mCherry Thr106. In the LaM4mCherry complex, the main interactions were in the CDR3 region of LaM4. There was a strong hydrogen bond interaction between Asn103 and mCherry Lys84 (2.07 ̊). There were also strong hydrogen bonds between Asn108 and mCherry Glu10 (2.00 ̊̊), Gly102 and mCherry Tyr38 (2.02 Å), and Leu101 and mCherry Asp81 (2.13 $)$ ). There was also a hydrogen bond between CDR1 Arg28 and mCherry Gln188 (2.16 ̊).

\section{Validation of the thermodynamics and binding affinity of the nanobody to mCherry by site-directed mutagenesis}

To further clarify the detailed driving forces of the binding between the Nbs and mCherry, we performed structurally guided site-directed mutagenesis and studied the binding affinity of the mutated Nbs to mCherry. We first used isothermal titration calorimetry (ITC) to measure the binding affinity and thermodynamic parameters because it is a label-free and in-solution method and is regarded as the gold standard for protein-protein interactions (Figure 3 and Table 2). Both LaM2 and LaM4 showed high binding affinity to mCherry; the Kd of LaM2-mCherry was $3.02 \mathrm{nM}$, and that of LaM4-mCherry was $22.5 \mathrm{nM}$ (Figure 3A and 3B). Then, we mutated some residues that contributed to the binding of mCherry. When the two residues of LaM2 CDR1 (Ser44) and CDR2 (Ser68) that form weak hydrogen bonds with mCherry were individually replaced by Ala, the binding affinity with mCherry was only slightly reduced (Figure 3A). However, when CDR3 Trp119 and Tyr120 were replaced by Ala simultaneously, the binding with mCherry was totally abolished (Figure $3 \mathrm{~A}$ ), indicating that this region was crucial for mCherry binding. In the LaM4-mCherry complex, replacement of CDR3 Asn104 with Lys, which reversed the charge, also completely abolished the binding to mCherry, indicating that the strong salt bridge between CDR3 Asn104 and mCherry Lys84 was crucial for the binding between LaM4 and mCherry. The replacement of Asn109 with Lys only slightly decreased the mCherry binding (Figure 3B).

\section{Validation of the simultaneous binding of LaM2 and LaM4 to mCherry}

The crystal structure of the LaM2-mCherry and LaM4-mCherry complexes showed that the binding regions of LaM2 and LaM4 to mCherry did not overlap, so we assumed that LaM2 and LaM4 could bind to mCherry simultaneously. We confirmed this assumption by ternary ITC and F-SEC experiments. The Kd of LaM2 titrated into the LaM4-

mCherry complex obtained by gel filtration was similar to that obtained for LaM2 directly titrated into mCherry (Figure 4A and Table 3). Additionally, the Kd of LaM4 titrated into the LaM2-mCherry complex is similar to that of titration into mCherry alone, indicating that the binding of LaM2 with mCherry and the binding of LaM4 with mCherry do not significantly affect each other. 
We also observed the formation of a ternary complex of LaM2-LaM4-mCherry by fluorescence-based size exclusion chromatography (F-SEC) [32], which can directly show the size of the biological macromolecule complex under physiological conditions. The F-SEC results also confirmed that a stable complex of 1:1:1 LaM2-LaM4-mCherry, 1:1 LaM2-mCherry and 1:1 LaM4-mCherry formed if the proper ratio of these proteins was mixed. It is worth noting that although LaM2 and LaM4 are similar in size, there was a certain difference in the position of the peak after binding with mCherry, which may be due to the different 3D shapes of the LaM2-mCherry and LaM4-mCherry complexes.

We also determined the size distributions of mCherry alone, LaM2-mCherry, LaM4-mCherry and LaM2-LaM4-mCherry by dynamic light scattering (DLS) experiments. The results showed that mCherry alone showed very high uniformity, centered at approximately $6 \mathrm{~nm}$, and formed complexes with the respective $\mathrm{Nbs}$; the size increased to approximately 11-12 nm (Figure 5A).

In contrast to some GFP Nbs (GFP enhancer and minimizer) [16], the binding of Nbs LaM2 and LaM4 does not significantly affect the chromophore environment of mCherry, resulting in little effect on the fluorescence properties of mCherry (Figure 5B). This feature ensured that the optical activity of mCherry would not change significantly with the binding of LaM2/LaM4; thus, quantification by RFP fluorescence remains accurate when manipulated through the binding of LaM2/LaM4 chimeric operators.

\section{Discussion}

The ITC results of LaM2 and LaM4 binding also provided thermodynamic information. For LaM2-mCherry binding, the total $\triangle \mathrm{G}$ change is $-19.85 \mathrm{kcal} / \mathrm{mol}$, the main energy contribution is the change in enthalpy $(\triangle \mathrm{H}-20.62 \mathrm{kcal} / \mathrm{mol})$, and the entropy change $-T \triangle S$ is $0.78 \mathrm{kcal} / \mathrm{mol}$. For LaM4-mCherry binding, the total $\triangle \mathrm{G}$ change is $-5.39 \mathrm{kcal} / \mathrm{mol}$, which is smaller than that of LaM2-mCherry. Both the enthalpy change $(-4.93 \mathrm{kcal} / \mathrm{mol})$ and entropy change $-\mathrm{T} \triangle S$ $(-0.46 \mathrm{kcal} / \mathrm{mol})$ contribute to the spontaneous formation of the LaM4-mCherry complex. Compared with LaM4, LaM2-mCherry binding is more specific because the enthalpy change is much more significant. If an application requires only one $\mathrm{Nb}$, LaM2 is a better candidate than LaM4. LaM4 binding is mainly driven by hydrophobic interactions, which are relatively nonspecific compared to the hydrogen bonds and salt bridge involved in LaM2 binding. Further optimization of LaM4 may be performed to replace the entropy-driven hydrophobic interaction by designing novel specific hydrogen bonds to mCherry residues.

While the molecular weight of a Nb is only approximately one tenth that of IgG, Nbs still provide a relatively large binding interaction interface. We calculated and compared the buried surface areas of LaM2 and LaM4 to mCherry and 3 Nbs of GFP (GBP1 enhancer PDB ID: 3K1K, GBP4 minimizer PDB ID: 3G9A and LaG16, PDB ID: 6LR7) to GFP, in addition to a representative PD-L1 Nb KN035 (PDB ID: 5JDS) entering clinical trials, by PISA [11]. All of these complexes have similar buried surface areas of approximately $600-700 \AA^{2}$, which is comparable to that of IgG and provides high affinity and specificity. We also compared the buried surface areas of 2 haptane Nbs (CorNb-Cortisone, PDB ID: 6ITQ [33] and MTX Nb-MTX, PDB ID: 3QXV [34]). Since these haptane antigens are relatively small and cannot provide a large surface for binding, the buried surface areas are relatively small, between 300-400 $\AA^{2}$; however, in contrast to small buried surface areas of protein antigens, over $50 \%$ of the haptane total surface is buried, showing the effectiveness of their interactions with specific antigens.

In addition to the delivery of plasmids encoding $\mathrm{Nbs}$, unlike IgG, $\mathrm{Nbs}$ can easily enter the cell membrane through a nonendocytic delivery system using a poly-Arg tag [20] and thus may have additional advantages over IgG-based chimeric manipulation systems. 
Simulations based on the crystal structures show that LaM4 can bind to the DsRed tetramer, and the binding sites are not on the DsRed self-multimerization interface; thus, the binding of LaM4 does not affect the tetramerization. Therefore, it is possible to design chimeric proteins linking functional operation components with LaM4 and develop a self-assembling macromolecular machine based on the RFP tetramer.

\section{Conclusion}

In summary, we have obtained the details of how Nbs LaM2 and LaM4 bind to mCherry's different epitopes at atomic resolution by structural biology. Additionally, our thermodynamic and molecular biology assays verified the crucial residues for the Nb-RFP interaction. The binding of LaM2 or LaM4 did not significantly change the fluorescence of mCherry, which is important for fluorescence quantification assays. LaM2 and LaM4 can bind simultaneously to mCherry, which is crucial for recruiting multiple operation elements to the RFP. These results provide important basic information for the development of a LaM2/LaM4-based RFP manipulation system and provide strategies to further optimize the binding affinity of Nbs to RFP.

\section{Abbreviations}

FP: fluorescent protein

GFP: green fluorescent protein

RFP: red fluorescent protein

ITC: isothermal titration calorimetry

MST: microscale thermophoresis

DLS: dynamic light scattering

$\mathrm{Nb}$ : nanobody

\section{Declarations}

\section{Ethics approval and consent to participate}

Not applicable.

\section{Consent for publication}

Not applicable.

\section{Availability of data and materials}

The datasets generated during and/or analyzed during the current study are available from the corresponding author on reasonable request.

\section{Competing interests}

We declare that we have no conflicts of interest in the authorship or publication of this contribution. 


\section{Funding}

This work was supported by grants 32070939,91527305 and 82030106 from the National Natural Science Foundation of China, an innovative research team of high-level local universities in Shanghai and a key laboratory program of the Education Commission of Shanghai Municipality (ZDSYS14005).

\section{Authors' contributions}

YD conceived and supervised the study; ZW, LL, RH and RL performed protein purification, verification, crystallization, and crystal data analysis; ZW, PZ, YZ and SC performed the protein-protein interaction assay.

\section{Acknowledgments}

We thank Prof. M. Hattori of Fudan University for the help with the F-SEC experiment. This work was supported by grants 32070939, 91527305 and 82030106 from the National Natural Science Foundation of China, an innovative research team of high-level local universities in Shanghai and a key laboratory program of the Education Commission of Shanghai Municipality (ZDSYS14005).

\section{References}

1. Shaner NC, Campbell RE, Steinbach PA, Giepmans BN, Palmer AE, Tsien RY. Improved monomeric red, orange and yellow fluorescent proteins derived from Discosoma sp. red fluorescent protein. Nat Biotechnol. 2004;22(12):1567-72.

2. Shu X, Shaner NC, Yarbrough CA, Tsien RY, Remington SJ. Novel chromophores and buried charges control color in mFruits. Biochemistry. 2006;45(32):9639-47.

3. Shcherbakova DM, Subach OM, Verkhusha VV. Red fluorescent proteins: advanced imaging applications and future design. Angew Chem Int Ed Engl. 2012;51(43):10724-38.

4. Y.Tsien R: The Green Fluorescent Protein. Annu Rev Biochem 1998.

5. Zimmer M. Green Fluorescent Protein (GFP): Applications, Structure, and Related Photophysical Behavior. Chem Rev 2002.

6. C.Hamers-Casterman. Naturally occurring antibodies devoid of light chains. Nature 1993.

7. Leow CH, Fischer K, Leow CY, Cheng Q, Chuah C, McCarthy J. Single Domain Antibodies as New Biomarker Detectors. Diagnostics (Basel) 2017, 7(4).

8. Scully M, Cataland SR, Peyvandi F, Coppo P, Knöbl P, Kremer Hovinga JA, Metjian A, de la Rubia J, Pavenski K, Callewaert F, et al. Caplacizumab Treatment for Acquired Thrombotic Thrombocytopenic Purpura. N Engl J Med. 2019;380(4):335-46.

9. Zimmermann I, Egloff P, Hutter CA, Arnold FM, Stohler P, Bocquet N, Hug MN, Huber S, Siegrist M, Hetemann L, et al: Synthetic single domain antibodies for the conformational trapping of membrane proteins. Elife $2018,7$.

10. Zimmermann I, Egloff P, Hutter CAJ, Kuhn BT, Bräuer P, Newstead S, Dawson RJP, Geertsma ER, Seeger MA. Generation of synthetic nanobodies against delicate proteins. Nat Protoc. 2020;15(5):1707-41.

11. Zhang F, Wei H, Wang X, Bai Y, Wang P, Wu J, Jiang X, Wang Y, Cai H, Xu T, et al. Structural basis of a novel PD-L1 nanobody for immune checkpoint blockade. Cell Discov. 2017;3:17004.

12. Weiss RA, Verrips CT. Nanobodies that Neutralize HIV. Vaccines (Basel) 2019, 7(3). 
13. Huo J, Le Bas A, Ruza RR, Duyvesteyn HME, Mikolajek H, Malinauskas T, Tan TK, Rijal P, Dumoux M, Ward PN, et al. Neutralizing nanobodies bind SARS-CoV-2 spike RBD and block interaction with ACE2. Nat Struct Mol Biol. 2020;27(9):846-54.

14. Schoof M, Faust B, Saunders RA, Sangwan S, Rezelj V, Hoppe N, Boone M, Billesbølle CB, Puchades C, Azumaya $\mathrm{CM}$, et al. An ultrapotent synthetic nanobody neutralizes SARS-CoV-2 by stabilizing inactive Spike. Science. 2020;370(6523):1473-9.

15. Xiang Y, Nambulli S, Xiao Z, Liu H, Sang Z, Duprex WP, Schneidman-Duhovny D, Zhang C, Shi Y. Versatile and multivalent nanobodies efficiently neutralize SARS-CoV-2. Science. 2020;370(6523):1479-84.

16. Kirchhofer A, Helma J, Schmidthals K, Frauer C, Cui S, Karcher A, Pellis M, Muyldermans S, Casas-Delucchi CS, Cardoso MC, et al. Modulation of protein properties in living cells using nanobodies. Nat Struct Mol Biol. 2010;17(1):133-8.

17. Zhang Z, Wang Y, Ding Y, Hattori M. Structure-based engineering of anti-GFP nanobody tandems as ultra-highaffinity reagents for purification. Sci Rep. 2020;10(1):6239.

18. Tang JC, Szikra T, Kozorovitskiy Y, Teixiera M, Sabatini BL, Roska B, Cepko CL. A nanobody-based system using fluorescent proteins as scaffolds for cell-specific gene manipulation. Cell. 2013;154(4):928-39.

19. Tang JC, Rudolph S, Dhande OS, Abraira VE, Choi S, Lapan SW, Drew IR, Drokhlyansky E, Huberman AD, Regehr WG, et al. Cell type-specific manipulation with GFP-dependent Cre recombinase. Nat Neurosci. 2015;18(9):133441.

20. Herce HD, Schumacher D, Schneider AFL, Ludwig AK, Mann FA, Fillies M, Kasper MA, Reinke S, Krause E, Leonhardt $\mathrm{H}$, et al. Cell-permeable nanobodies for targeted immunolabelling and antigen manipulation in living cells. Nat Chem. 2017;9(8):762-71.

21. Simpson LM, Macartney TJ, Nardin A, Fulcher LJ, Röth S, Testa A, Maniaci C, Ciulli A, Ganley IG, Sapkota GP. Inducible Degradation of Target Proteins through a Tractable Affinity-Directed Protein Missile System. Cell Chem Biol. 2020;27(9):1164-80.e1165.

22. Fridy PC, Li Y, Keegan S, Thompson MK, Nudelman I, Scheid JF, Oeffinger M, Nussenzweig MC, Fenyö D, Chait BT, et al. A robust pipeline for rapid production of versatile nanobody repertoires. Nat Methods. 2014;11(12):125360.

23. Prole DL, Taylor CW. A genetically encoded toolkit of functionalized nanobodies against fluorescent proteins for visualizing and manipulating intracellular signalling. BMC Biol. 2019;17(1):41.

24. Wang Q-S, Zhang K-H, Cui Y, Wang Z-J, Pan Q-Y, Liu K, Sun B, Zhou H, Li M-J, Xu Q, et al. Upgrade of macromolecular crystallography beamline BL17U1 at SSRF. Nucl Sci Tech. 2018;29(5):68.

25. Zhang W-Z, Tang J-C, Wang S-S, Wang Z-J, Qin W-M, He J-H. The protein complex crystallography beamline (BL19U1) at the Shanghai Synchrotron Radiation Facility. Nucl Sci Tech. 2019;30(11):170.

26. Otwinowski Z, Minor W. Processing of X-ray diffraction data. Methods enzymol. 1997;276:307-26.

27. Vagin A, Teplyakov A. MOLREP: an automated program for molecular replacement. J Appl Crystallogr. 1997;30:1022-5.

28. Vagin AA, Steiner RA, Lebedev AA, Potterton L, McNicholas S, Long F, Murshudov GN. REFMAC5 dictionary: organization of prior chemical knowledge and guidelines for its use. Acta Crystallogr D. 2004;60:2184-95.

29. Adams PD, Afonine PV, Bunkoczi G, Chen VB, Davis IW, Echols N, Headd JJ, Hung LW, Kapral GJ, GrosseKunstleve RW, et al. PHENIX: a comprehensive Python-based system for macromolecular structure solution. Acta crystallographica Section D Biological crystallography. 2010;66(Pt 2):213-21. 
30. Emsley P, Lohkamp B, Scott WG, Cowtan K. Features and development of Coot. Acta Crystallogr D. 2010;66:486501.

31. DeLano WL, Lam JW. PyMOL: A communications tool for computational models. Abstr Pap Am Chem S. 2005;230:U1371-2.

32. Hattori M, Hibbs RE, Gouaux E. A fluorescence-detection size-exclusion chromatography-based thermostability assay for membrane protein precrystallization screening. Structure. 2012;20(8):1293-9.

33. Ding L, Wang Z, Zhong P, Jiang H, Zhao Z, Zhang Y, Ren Z, Ding Y. Structural insights into the mechanism of single domain VHH antibody binding to cortisol. FEBS Lett. 2019;593(11):1248-56.

34. Fanning SW, Horn JR. An anti-hapten camelid antibody reveals a cryptic binding site with significant energetic contributions from a nonhypervariable loop. Protein Sci. 2011;20(7):1196-207.

\section{Tables}

Table 1 Data collection and refinement statistics. The values in parentheses are for the high-resolution shells.

\begin{tabular}{|c|c|c|}
\hline & LaM2-mCherry & LaM4-mCherry \\
\hline PDB entry & 6IR2 & 6IR1 \\
\hline Space group & P 212121 & C 121 \\
\hline$a, b, c(\AA)$ & 49.81761 .046109 .21 & 156.48941 .66553 .182 \\
\hline$a, \beta, y\left({ }^{\circ}\right)$ & 909090 & 9090.62890 \\
\hline Resolution $(\AA)$ & 29.4-1.393 (1.443-1.393) & $32.55-1.919$ (1.987-1.919) \\
\hline $\mathrm{l} / \delta(\mathrm{I})$ & $7.5(2.3)$ & $8.0(3.0)$ \\
\hline Completeness (\%) & $97.51(93.96)$ & $92.93(90.98)$ \\
\hline Number of unique reflections & $65713(6230)$ & $24709(2411)$ \\
\hline Rwork & $0.1950(0.2682)$ & $0.1799(0.2581)$ \\
\hline Rfree & $0.2157(0.2935)$ & $0.2319(0.3110)$ \\
\hline \multicolumn{3}{|l|}{ RMSD } \\
\hline Bond length $(\AA)$ & 0.006 & 0.013 \\
\hline Bond angles $\left({ }^{\circ}\right)$ & 1.34 & 1.51 \\
\hline Average $B$, all atoms $\left(\AA^{2}\right)$ & 20.23 & 31.98 \\
\hline
\end{tabular}

Table 2 The binding specificity of LaM2 and LaM4 series nanobodies to mCherry by ITC 


\begin{tabular}{|c|c|c|c|c|c|c|}
\hline & $\mathbf{n}$ & $\Delta \mathrm{H}^{\circ} \mathrm{Okcal} / \mathrm{mol} \mathrm{l}$ & $\Delta \mathrm{S} \square \mathrm{cal} / \mathrm{mol} / \mathrm{deg} \square$ & $\begin{array}{l}-\mathrm{T} \Delta \mathrm{S}^{\circ} \\
(\mathrm{kcal} / \mathrm{mol})\end{array}$ & $\stackrel{\mathrm{AG}^{\circ}}{(\mathrm{kcal} / \mathrm{mol})}$ & $\mathrm{K}_{\mathrm{D}}(\mathrm{nM})$ \\
\hline LaM2 & $0.83 \pm 0.01$ & $-20.62 \pm 0.17$ & -30.1 & 0.78 & $-19.85 \pm 0.17$ & $3.02 \pm 1.88$ \\
\hline LaM2-S44A & $0.94 \pm 0.01$ & $-17.60 \pm 0.15$ & -21.8 & 0.55 & $-17.05 \pm 0.15$ & $7.19 \pm 2.45$ \\
\hline LaM2-S68A & $1.19 \pm 0.01$ & $-15.60 \pm 0.09$ & -17.4 & 0.44 & $-15.16 \pm 0.09$ & $23.64 \pm 3.94$ \\
\hline $\begin{array}{l}\text { LaM2- } \\
\text { W119AY120A }\end{array}$ & - & - & - & - & - & - \\
\hline LaM4 & $0.69 \pm 0.01$ & $-4.93 \pm 0.15$ & 18.5 & -0.46 & $-5.39 \pm 0.15$ & $22.50 \pm 34.60$ \\
\hline LaM4-N104K & - & - & - & - & - & - \\
\hline LaM4-N109K & $0.74 \pm 0.02$ & $-2.89 \pm 0.09$ & 21.8 & -0.55 & $-3.43 \pm 0.09$ & $132.27 \pm 63.59$ \\
\hline
\end{tabular}

Table 3 The binding specificity of LaM2 to the LaM4-mCherry complex and LaM4 to the LaM2-mCherry complex by ITC

\begin{tabular}{|c|c|c|c|c|c|c|}
\hline & $\mathrm{n}$ & $\triangle \mathrm{H}^{\circ} \mathrm{kccal} / \mathrm{mol}[$ & $\Delta \mathrm{S} \square \mathrm{cal} / \mathrm{mol} / \mathrm{deg} \square$ & $\begin{array}{l}-\mathrm{T} \Delta \mathrm{S}^{\circ} \\
(\mathrm{kcal} / \mathrm{mol})\end{array}$ & $\begin{array}{l}\mathrm{AG}^{\circ} \\
(\mathrm{kcal} / \mathrm{mol})\end{array}$ & $\mathrm{K}_{\mathrm{D}}(\mathrm{nM})$ \\
\hline $\begin{array}{l}\text { LaM2 to } \\
\text { LaM4- } \\
\text { mCherry }\end{array}$ & $0.866 \pm 0.003$ & $-22.14 \pm 0.15$ & -37.3 & 0.93 & $-21.21 \pm 0.15$ & $8.33 \pm 1.27$ \\
\hline $\begin{array}{l}\text { LaM4 to } \\
\text { LaM2- } \\
\text { mCherry }\end{array}$ & $1.010 \pm 0.036$ & $-1.99 \pm 0.10$ & 23.8 & -0.60 & $2.59 \pm 0.10$ & $216.40 \pm 92.28$ \\
\hline
\end{tabular}

Table 4 Comparison of typical buried surface areas after specific nanobody binding to their antigens

\begin{tabular}{|lll|}
\hline Interaction & Buried Surface $\left(\AA^{2}\right)$ & PDB ID \\
\hline LaM2-mCherry & 679.6 & $6 I R 2$ \\
\hline LaM4-mCherry & 757.8 & $6 I R 1$ \\
\hline GBP1(enhancer)-GFP & 681.5 & $3 \mathrm{~K} 1 \mathrm{~K}$ \\
\hline GBP4(minimizer)-GFP & 651.9 & $3 \mathrm{G} 9 \mathrm{~A}$ \\
\hline LaG16-GFPuv & 700.0 & 6 LR7 \\
\hline KN035-PD-L1 & 626.2 & $5 \mathrm{JDS}$ \\
\hline CorNb-Cortisone & 337.9 & $6 I T Q$ \\
\hline MTX Nb-MTX & 433.9 & $3 \mathrm{QXV}$ \\
\hline
\end{tabular}




\section{Figures}

A

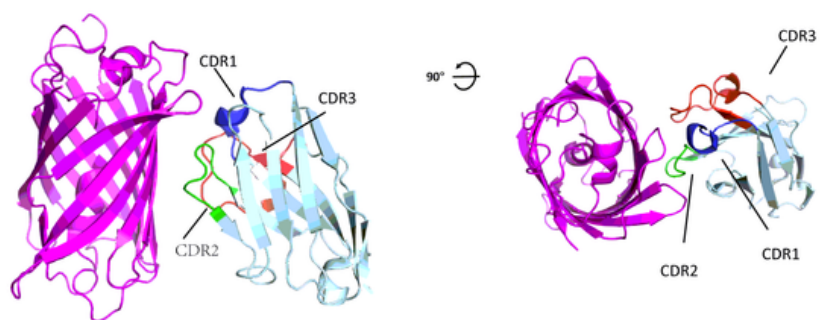

B
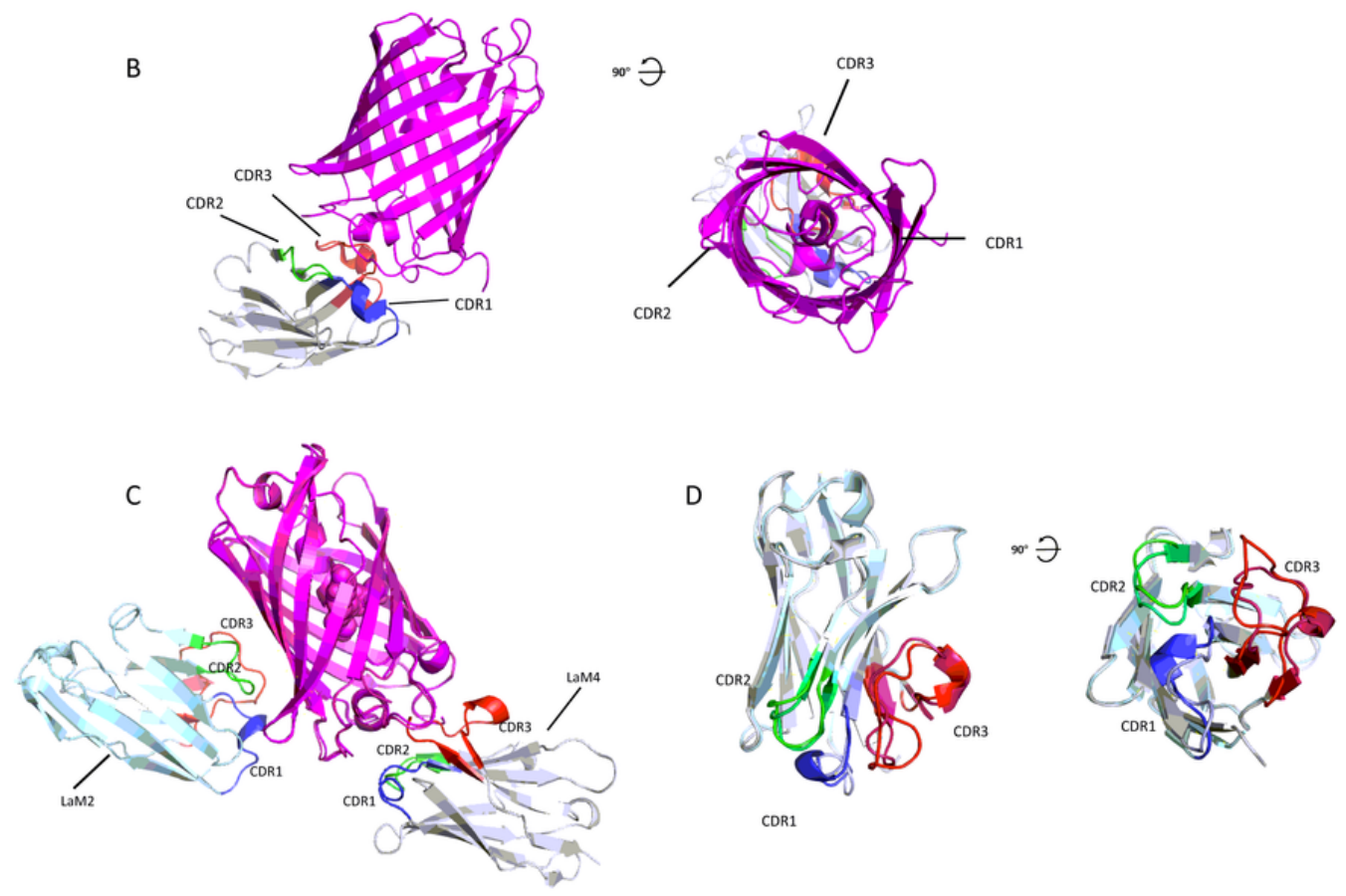

E
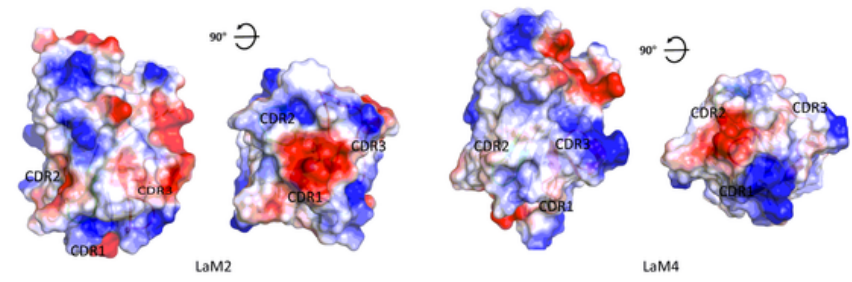

\section{Figure 1}

Structure of mCherry: mCherry-specific nanobody complex determined by X-ray crystallography. A. Overall structure of LaM2-mCherry complex. mCherry is shown in purple. LaM2 is shown in light blue, and CDR1-3s are shown in blue, green and red (PDB ID: 6IR2). B. Overall structure of the LaM4-mCherry complex. mCherry is shown in purple. LaM4 is shown in gray, and the CDRs are shown in blue (CDR1), green (CDR2) and red (CDR3) (PDB ID: 6IR1). C. Alignment of LaM2 (light blue)-mCherry and LaM4 (gray)-mCherry complex. D. Alignment of Nbs LaM2 and LaM4. E. Comparison of the surface potential of Nbs LaM2 and LaM4 (blue: positive, red: negative). 
A
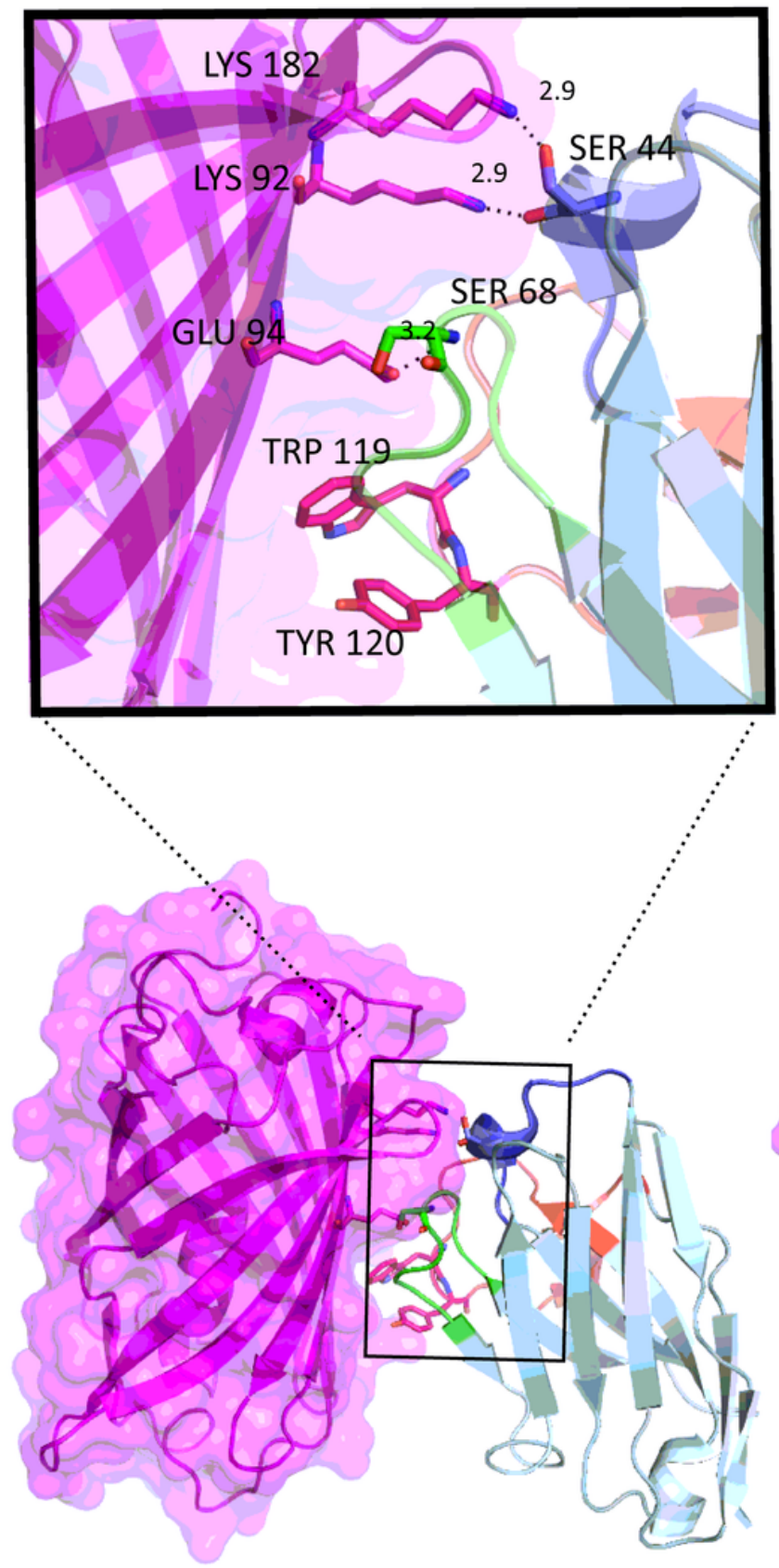

$\mathrm{B}$
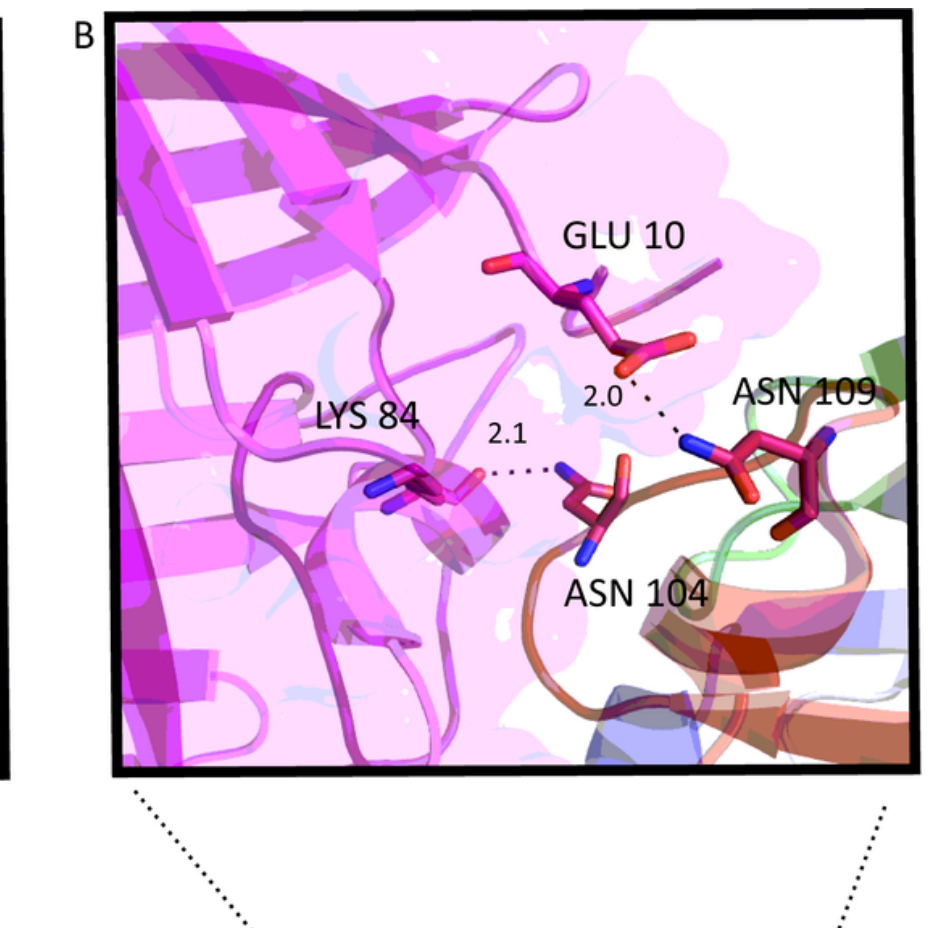

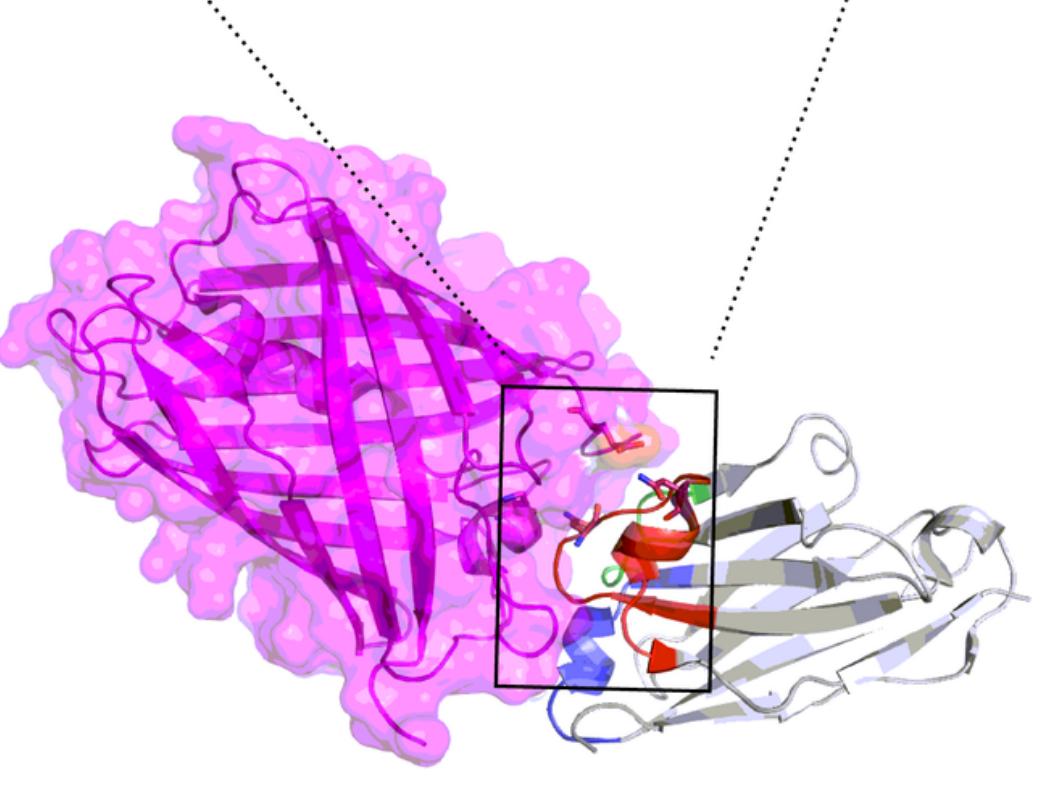

Figure 2

The detailed binding interfaces of Nbs to mCherry. A. The detailed interactions of LaM2 CDRs with mCherry. B. The detailed interactions of LaM4 CDRs with mCherry. 
A

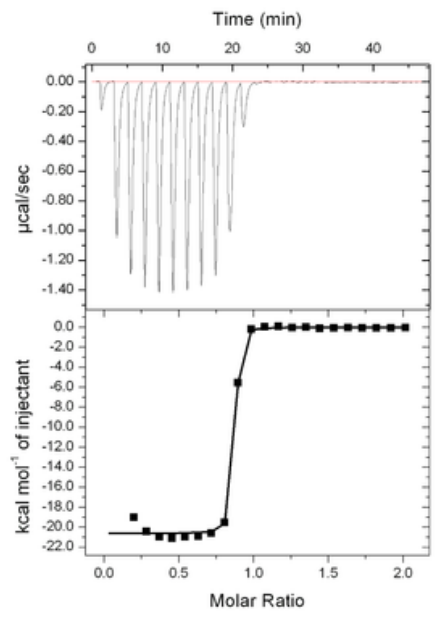

LaM2

B

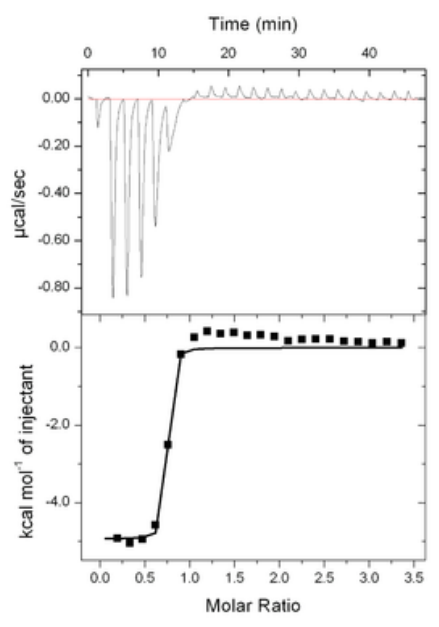

LaM4

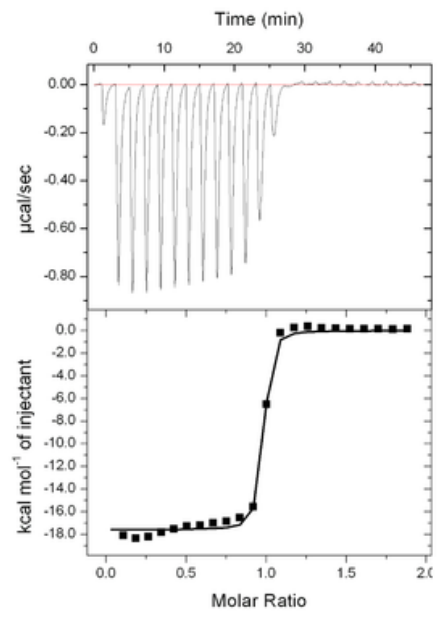

LaM2 S44A

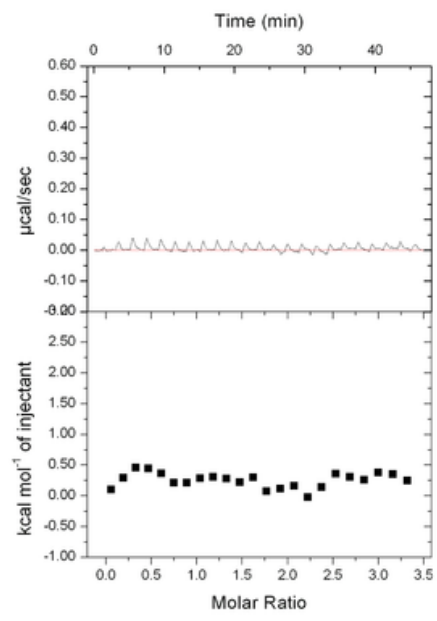

LaM4 N104K

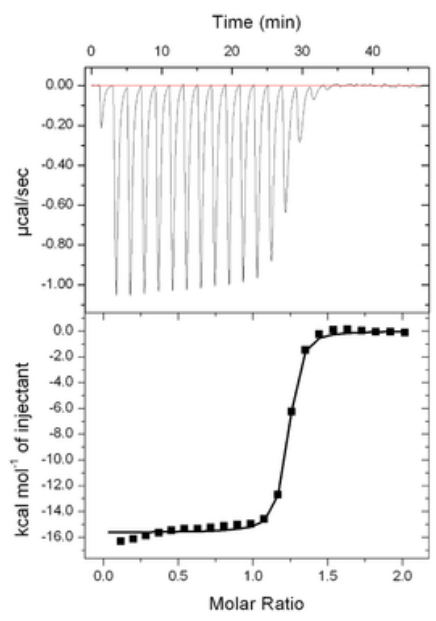

LaM2 S68A

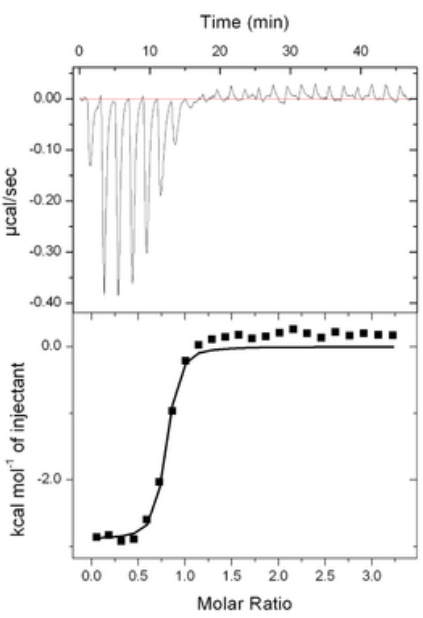

LaM4 N109K

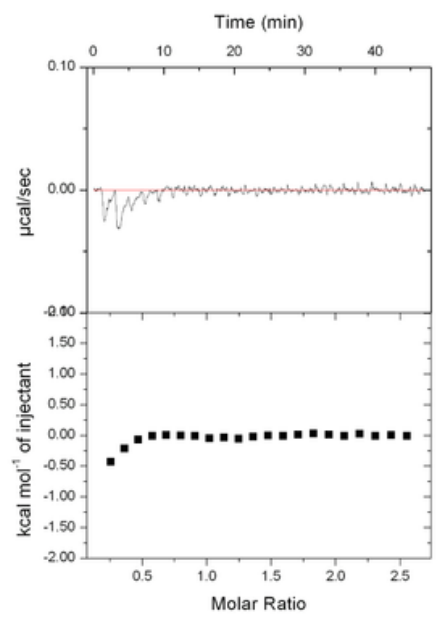

LaM2 W119A_Y120A

\section{Figure 3}

Validation of the interaction between nanobodies (including site-directed mutants) and mCherry by isothermal titration calorimetry at $25^{\circ} \mathrm{C}$. A. LaM2 series nanobodies (wild type, S44A, S68A and W119A/Y120A); B. LaM4 series nanobodies (wild type, N104K and N109K). 
A

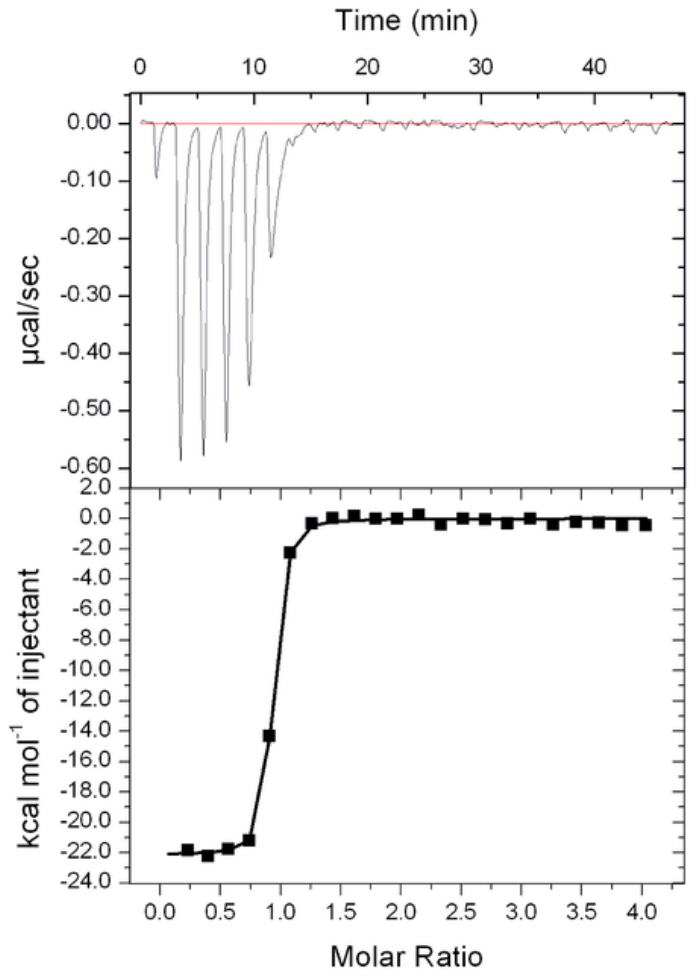

LaM2 to mCherry-LaM4 complex

C

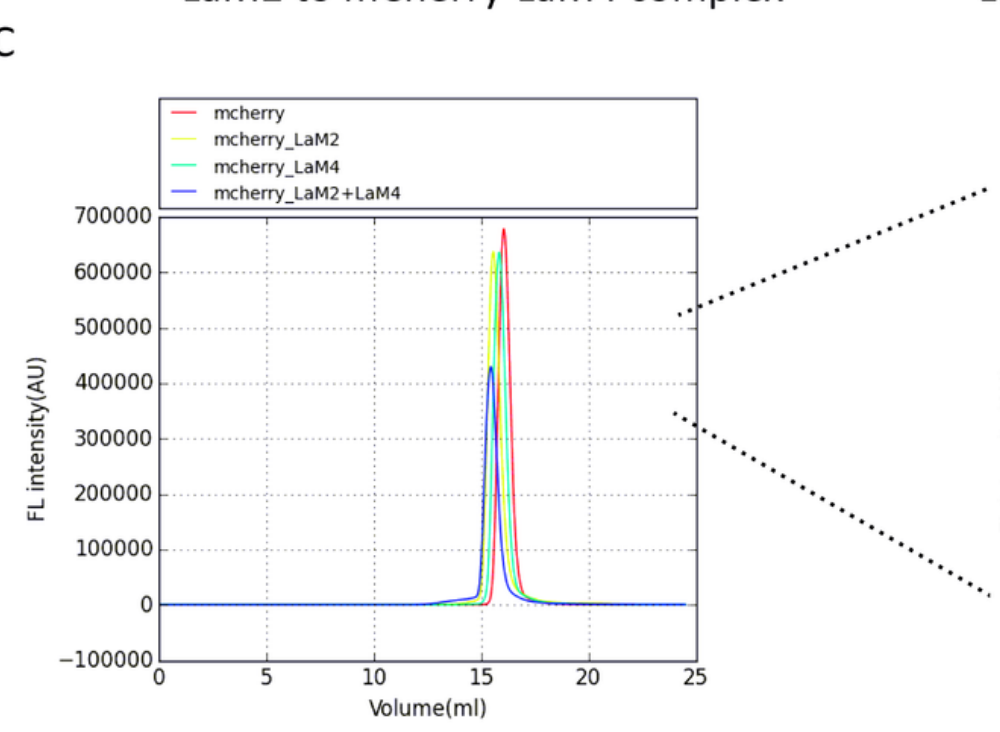

B Time (min)

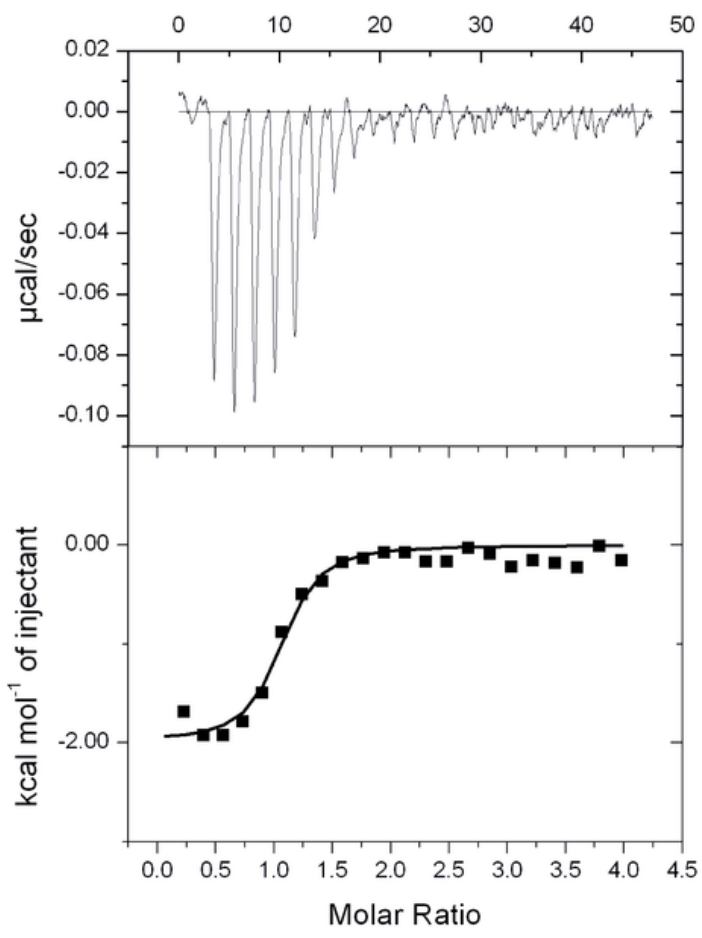

LaM4 to mCherry-LaM2 complex

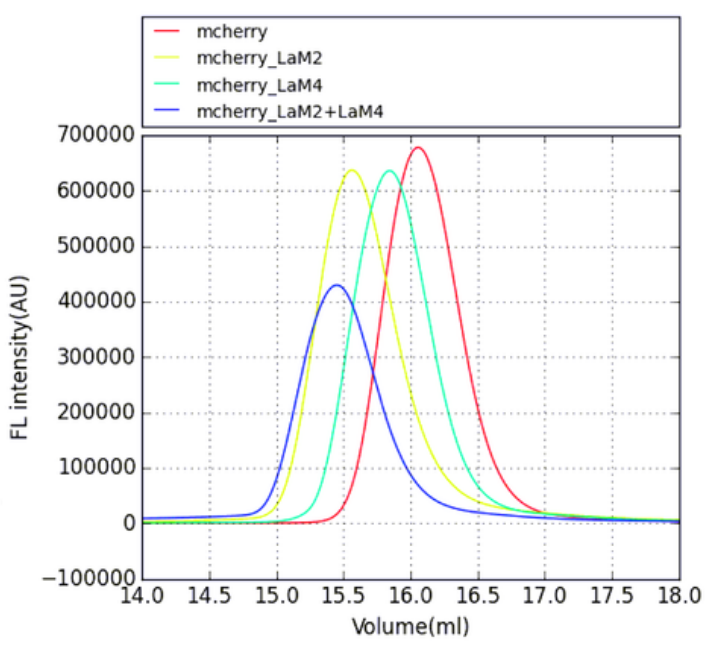

Figure 4

ITC and F-SEC assays confirmed that LaM2 and LaM4 can bind simultaneously to mCherry. A. The ITC result of LaM2 binding to the mCherry-LaM4 preformed complex. B. The ITC result of LaM4 binding to the mCherry-LaM2 preformed complex. C. The F-SEC results of mCherry (red), mCherry-LaM2 (light green), mCherry-LaM4 (dark green) and the premixed mCherry-LaM2 complex mixed with LaM4 (blue). The right panel shows an enlargement of 14-17 $\mathrm{ml}$ of F-SEC to clearly see the change in size. 
A

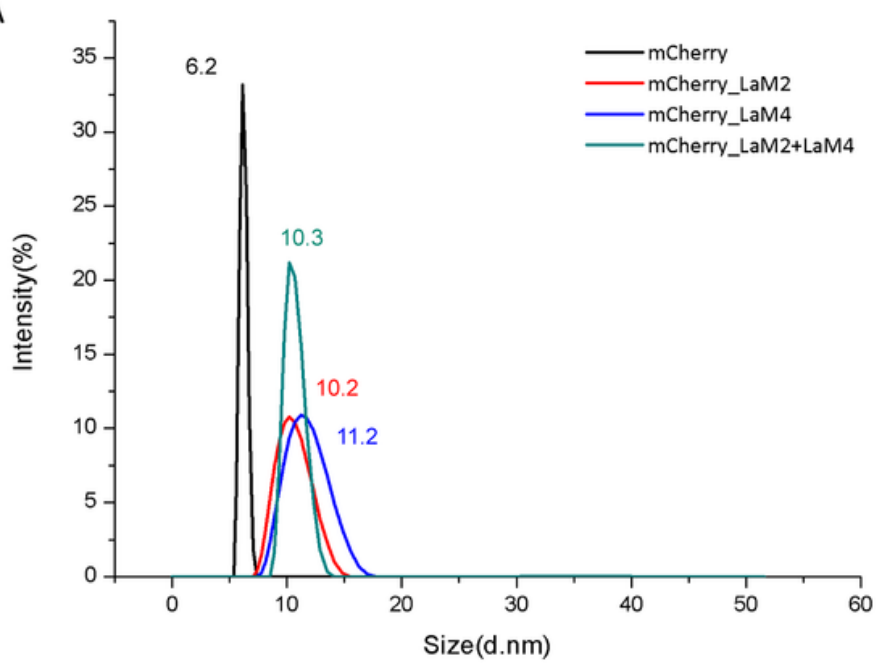

B

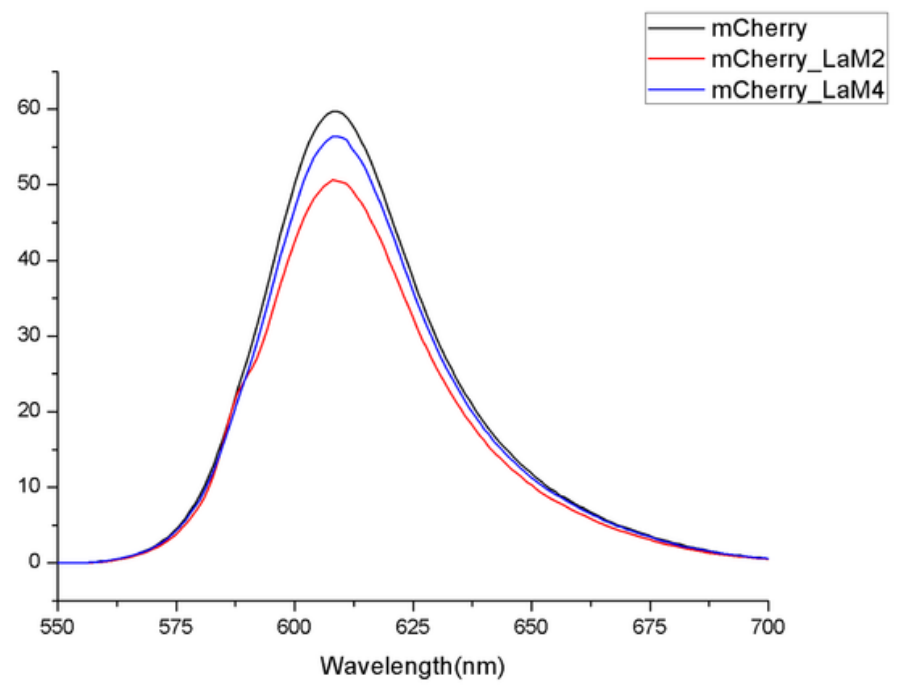

Figure 5

The size and fluorescence intensity of mCherry binding with nanobodies. A. The size distributions of mCherry (black), LaM2-mCherry (red), LaM4-mCherry (blue) and LaM2-mCherry-LaM4 (green) by dynamic light scattering. B. The emission spectra of mCherry (black), LaM2-mCherry (red) and LaM4-mCherry (blue).

\section{Supplementary Files}

This is a list of supplementary files associated with this preprint. Click to download.

- mCherrySuppl20210402.docx 